\title{
1 Immunostimulatory guide RNAs mediate potent antiviral response
}

2

3 Yujia Cai ${ }^{1,2 *}$, Alice Knudsen ${ }^{2}$, Samuel Joseph Windross ${ }^{2}$, Martin K Thomsen ${ }^{2}$ and Søren R Paludan ${ }^{2 *}$

$4{ }^{1}$ Key Laboratory of Systems Biomedicine, Shanghai Center for Systems Biomedicine, Shanghai Jiao 5 Tong University, Shanghai 200240, China.

$6 \quad{ }^{2}$ Department of Biomedicine, Aarhus University, Aarhus C, DK-8000, Denmark.

$7 \quad{ }^{*}$ For correspondence: yujia.cai@sjtu.edu.cn; srp@biomed.au.dk

8

$9 \quad$ Running title: Immune stimulating gRNAs suppress virus

10

11 Keywords: herpes simplex virus 1, gRNA, innate immune response, CRISPR, antivirus 


\section{Abstract}

2 Genome-editing with CRISPR has emerged as a technology with broad therapeutic potential.

3 However, it is unclear whether CRISPR will elicit innate immune responses, which could impact both

4 positively and negatively on the desired therapeutic effects. Here, we have examined the immune-

5 stimulatory properties of different variants of guide RNAs (gRNAs) - in vitro transcribed gRNA

6 (IVT-gRNA) and synthetic gRNAs with or without chemical modifications, full-length or duplexed.

7 We find that only IVT-gRNA evokes strong expression of cytokines in a panel of cell lines while all

8 the synthetic RNAs do not. We further find that sensing of IVT-gRNA proceeds mainly through the RIG-I/MAVS RNA sensing axis. One potential use of CRISPR is for antiviral therapy. The antiviral actions of the gRNA tested up until now have been relying purely on the gene editing function of the CRISPR machinery, which weakens its feasibility due to the difficulty to target all infected cells. When IVT-gRNA was combined with unmodified Cas9 mRNA, which also induces cytokine expression, strong immune response was obtained while maintaining nuclease activity of CRISPR. Remarkably, such combination inhibited herpes simplex virus type-1 (HSV-1) replication even though the nuclease activity was modest, and provided 'bystander protection' to the cells that were not transfected with CRIPSR molecules. The antiviral activity of IVT-gRNA was also observed in vivo in HSV-1-infected Cas9+ mice, thus demonstrating the therapeutic potential. Our study further extends the applications of CRISPR by exploiting the immunostimulatory function of gRNAs. 


\section{Introduction}

CRISPR is now broadly used in basic and translational research. The technology was originally invented for its site-specific nuclease activity (Jinek et al. 2012; Cong et al. 2013; Jinek et al. 2013), and has subsequently been broadened to also allow transcription activation, nucleotide changing, and epigenetic reprograming (Maeder et al. 2013; Mali et al. 2013; Perez-Pinera et al. 2013; Komor et al. 2016; Vojta et al. 2016). For a successful clinical translation, understanding the host response to CRISPR is crucial as the potential immune reaction could conceivably lead to compromised gene editing efficiency and clearance of modified cells. It has been noted that long-term Cas9 exposure elicits Cas9 specific T-cell expansion and antibody production in mice (Chew et al. 2016). Prior to adaptive immune responses to Cas9 protein, the CRISPR can be sensed by the innate immune system which can detect foreign nucleic acids and is normally involved in recognition of invading viruses and bacteria. So far, it is unclear if gRNA - another component of CRISPR - will be recognized by the targeted cells. The gRNA which guides Cas9/gRNA complex to an intended genomic locus is a 100-nucleotides sequence consisting of both single- and double-stranded structures. It can be transcribed intracellularly driven by U6 promoter or can be delivered directly in the RNA format from in vitro transcription or synthesis. The in vitro transcribed gRNA (IVT-gRNA) together with Cas9 mRNA have been commonly used for microinjection into one-cell-stage embryos to quickly generate gene-modified animals (Niu et al. 2014). The synthetic gRNA with chemical modifications has shown superior efficiency and demonstrates potential in gene therapy research (Hendel et al. 2015; Dever et al. 2016). Compared to the well characterized nuclease activity, it is unclear if administration of gRNAs causes additional effects in the intracellular environment such as innate immune response. In this study, we investigated the innate immune stimulatory properties of gRNAs from different sources - in vitro transcribed gRNA and a variety of synthetic gRNAs. In several cell types, we found that IVT-gRNA, but none of the synthetic gRNAs, elicits strong expression of a panel of cytokines including IFNB1, TNFA, IL6 and CXCL10 while maintaining nuclease activity. Taking advantage of this dual function of IVT-gRNAs, we demonstrated that they induced highly efficient antiviral response and provided bystander protection to the neighbouring non-transfected cells. By using a HSV-1 infection mouse model, we further extend the robust antiviral effects of IVT-gRNA in vivo. 


\section{Results}

\section{In vitro transcribed - but not the synthetic gRNAs - induce strong cytokine responses}

Cells express a variety of pattern recognition receptors (PRRs), which sense danger signals through binding to microbial nucleic acids, proteins and carbohydrates (Mogensen 2009). The recognition of external substrates by PRRs initiates a hierarchical innate immune signalling leading to activation of specific gene expression programmes. In particular, intracellular nucleic-acid-sensing PRRs induce expression of type I and type III IFNs, which have strong antiviral activity (Mogensen 2009). To characterize the immunostimulatory properties of gRNAs, we first transfected THP1-derived macrophage-like cells with five types of gRNA targeting to the endogenous CCR5 locus. The gRNAs were in vitro transcribed gRNAs (IVT) and four synthetic variants of gRNAs, namely full-length unmodified (FL-UM) and modified (FL-MO) gRNAs, and duplexed unmodified (Du-UM) and modified (Du-MO) gRNAs. We then examined innate immune response by measuring expression of the cytokines, IFNB1, TNFA, CCL5, IL6, and CXCL10 as well-as the IFN-stimulated gene MX1. We found that IVT-gRNA induced robust cytokines expression to an extent comparable to the doublestranded RNA mimic poly(I:C), which is a known potent inducer of innate immune responses (Fig. 1A-F). The strong induction of IFNB1 expression by IVT-gRNA was also seen in HEK293T cells and the human plasmacytoid dendritic cell line pDC-gen2.2 (Fig. 1G-H). Notably, none of the synthetic gRNAs enhanced the expression of the genes examined - regardless the chemical modifications (Fig. 1A-H). The immune stealth property of synthetic gRNAs might make them a preferred choice for ex vivo gene therapy where limited immune activation is desired and to preserve the 'stemness' of hematopoietic stem cells (Piras et al. 2017; Takizawa et al. 2017). To test if innate immune activation by gRNAs was dependent on transfection reagent, we transfected gRNAs with 3 different cationic lipid or polymer reagents. IVT-gRNA preserved the ability to induce IFNBI expression while the modified synthetic gRNA did not evoke any significant cytokine expression with all the transfection reagents (Fig. 1I and Supplemental_Fig_S1A). As CRISPR nuclease function requires formation of gRNA and Cas9 complexes, we also evaluated the commonly used Cas9 sources - the modified and unmodified Cas9 mRNA. While unmodified Cas 9 mRNA clearly induced expression of cellular expression of immune genes, notably IFNB1 and TNFA, the modified Cas9 mRNA was immune silent (Supplemental_Fig_S2A-F). 
A

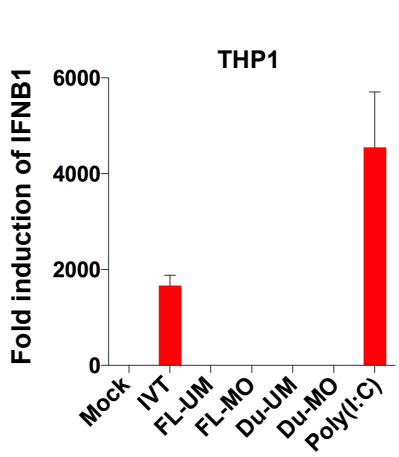

D

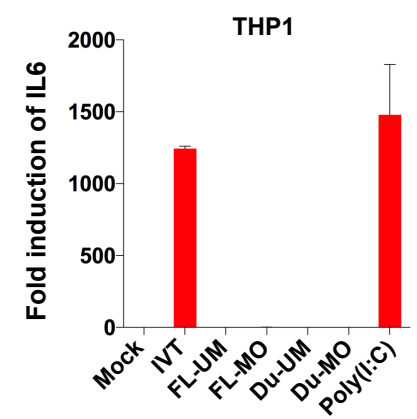

G

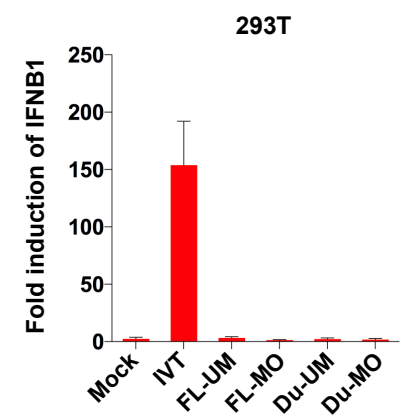

B

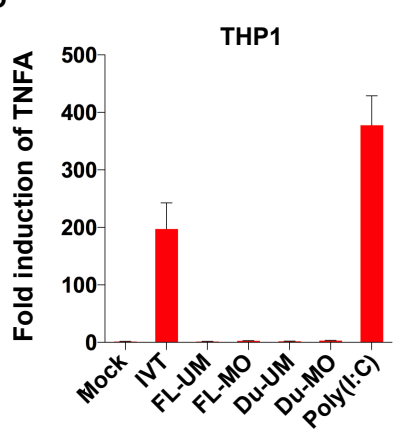

E

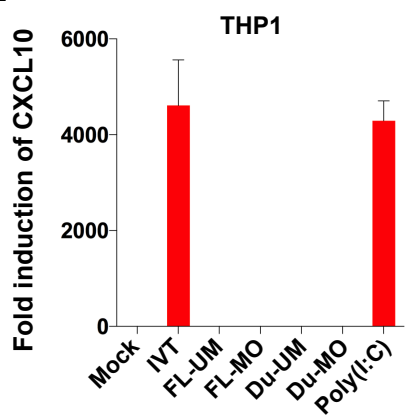

$\mathrm{H}$

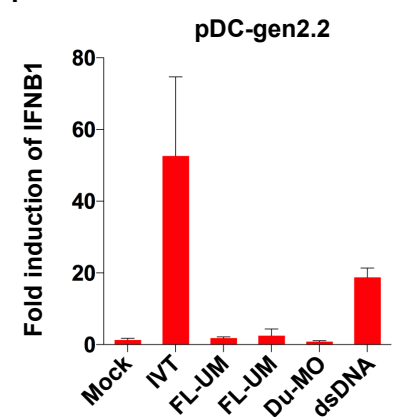

C

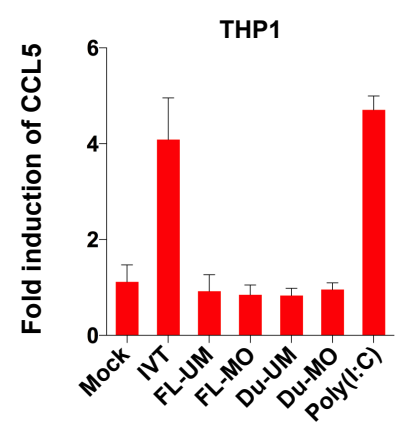

$\mathrm{F}$

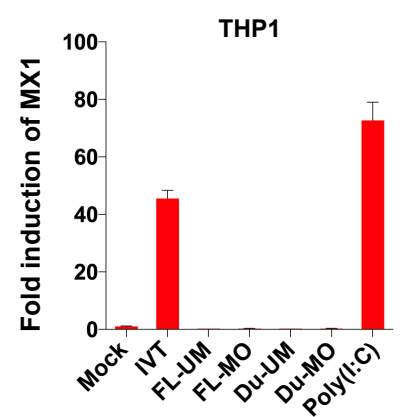

I

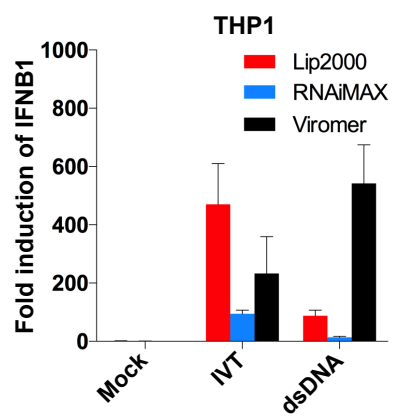

2 Fig. 1. The innate immune property of gRNA variants. (A-F) Fold induction of $I F N B 1, T N F A$,

3 CCL5, IL6, CXCL10 and MX1, respectively, by different variants of gRNA including in vitro

4 transcribed gRNA (IVT), chemically modified and unmodified full-length synthetic gRNA (FL-MO

5 and FL-UM), duplexed synthetic gRNA chemically modified (Du-MO) and unmodified (Du-UM) in

6 THP1 derived macrophages. Poly(I:C) serves as a control. (G) and (H) The immune inducing

7 capability of gRNA variants were verified in 293T cells and pDC-gen2.2 cells by measuring the fold

8 change of IFNB1. (I) Fold induction of IFNB 1 by IVT-gRNA in THP1 derived macrophages using

9 Lipofectamine 2000, RNAi/MAX and Viromer RED. DsDNA (60-mer) serves as a control. All gRNAs target to CCR5 locus. 
1 The sequence and secondary structure of a CCR5 locus targeting IVT-gRNA with a 5' triphosphate

2 (ppp) moiety is illustrated in Fig. 2A. We first investigated the mechanism of IVT-gRNA induced innate immune sensing using $293 \mathrm{~T}$ cell lines knockout of three cytoplasmic RNA sensors - retinoic acid-inducible gene-I (RIG-I), melanoma differentiation-associated gene 5 (MDA5) and protein kinase $\mathrm{R}(P K R)$. While knockout of MDA5 and PKR preserved IFNB1 induction, knockout of RIG-I completely abolished IVT-gRNA-induced IFNBI expression indicating RIG-I to be the primary sensor of IVT-gRNA (Fig. 2B). Using the same setting, we investigated mechanism to sense the unmodified Cas9 mRNA and found reduced IFNBI induction only when RIG-I was knockout (Supplemental_Fig_S3A). Next, we investigated which adaptor molecule for nucleic acids sensors was involved in the IVT-gRNA sensing pathway. We found that cells lacking mitochondrial antiviralsignaling protein (MAVS), but not cells lacking stimulator of IFN genes (STING) exhibited reduced capacity to evoke IFNB1 expression following IVT-gRNA transfection (Fig. 2C). RIG-I recognizes the biphosphate and triphosphate moieties in 5'-terminus of double or single stranded RNAs, AU- or polyU/UC-rich region, and specific secondary structure of viral genome (Hornung et al. 2006; Pichlmair et al. 2006; Goubau et al. 2014; Lassig et al. 2015; Sanchez David et al. 2016). Notably, cells knockout of TIR-domain-containing adapter-inducing IFN-beta (TRIF) were able to efficiently promote IFNBI production but significantly lower than the wild-type counterpart (Fig. 2D and Supplemental_Fig_S3B). To investigate the mechanism in IVT-gRNA sensing, we pre-treated the IVT-gRNA with Alkaline Phosphatase (CIP) to remove the 5' triphosphate group before transfection. This treatment reduced the induction of IFNB1 in THP1-derived macrophages (Fig. 2E). Furthermore, the reduced induction of IFNB1 was recapitulated when capped IVT-gRNA was used for transfection (Fig. 2E). As the IVT-gRNA can be assembled into ribonucleoprotein (RNP) with Cas9 protein in vitro, we therefore ask whether preassembly can minimize IVT-gRNA sensing. Indeed, we found significantly lower of IFNB1 production likely due to preassembly excluded the binding of RIG-I to gRNA (Fig. 2F). Together, these data suggest RIG-I recognition to the 5' triphosphate group but not the secondary RNA structure is the main contributor to IVT-gRNA induced immune sensing. 
A

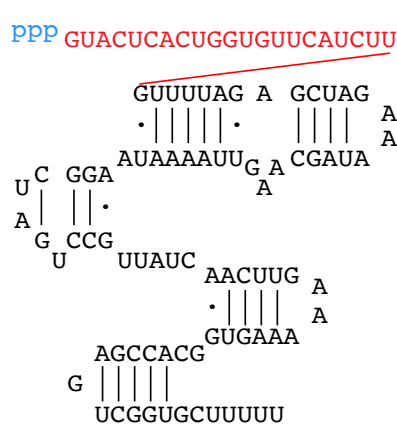

$\mathrm{D}$

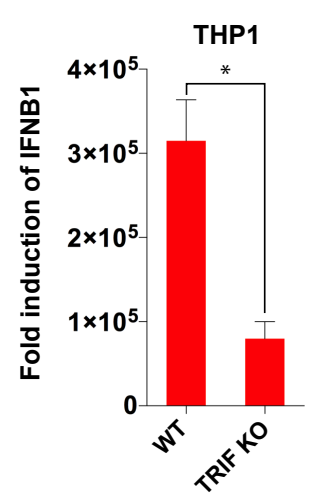

B

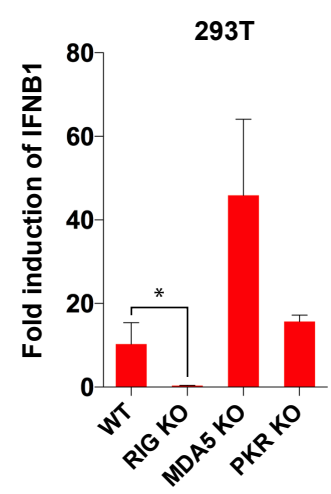

$E$

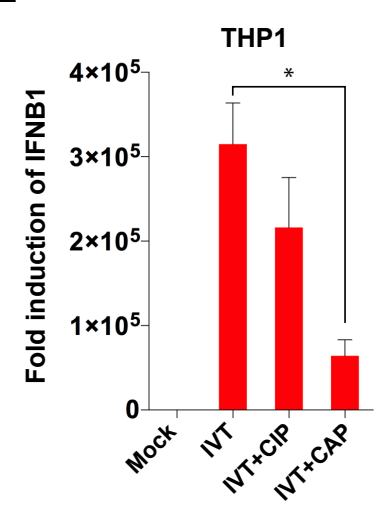

C

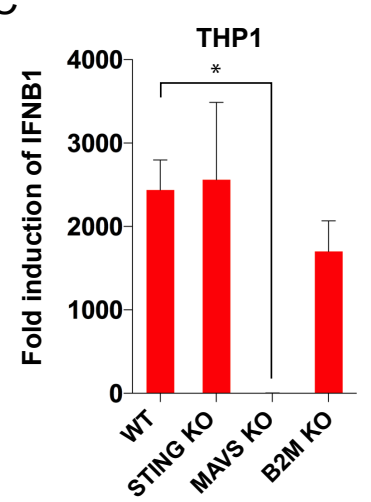

$\mathrm{F}$

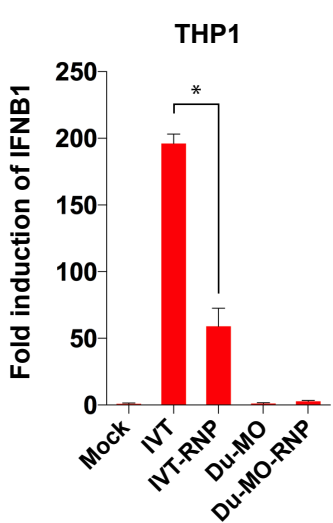

2 Fig. 2. The mechanism of IVT-gRNA induced innate immune response. (A) Sequence and secondary structure of in vitro transcribed gRNA targeting to CCR5 locus. The first three blue letters in lower case indicate triphosphate modification and the followed red letter in upper case is the CCR5locus targeting sequence. The black letter in upper case is backbone of IVT-gRNAs. (B) Evaluation of the innate immune induction by IVT-gRNA in 293 T cells knockout of RNA sensor RIG-I, MDA5 and $P K R$, respectively. (C) and (D) Examining the innate immune induction by IVT-gRNA in THP1 derived macrophages knockout of adaptor protein STING, MAVS and TRIF, respectively. B2M knockout line serves as a control. (E) Fold induction of IFNBI by IVT-gRNA, IVT-gRNA pre-treated with CIP and capped IVT-gRNA. (F) Fold induction of IFNBI by IVT-gRNA and IVT-gRNA assembled with recombinant Cas9 protein (RNP). The chemically modified duplexed synthetic gRNA and its Cas9 assembled format serve as negative controls.

\section{Gene disruption efficiency of gRNA variants}

To compare the genome-editing efficiency of different in vitro transcribed and synthetic gRNAs, we generated a $293 \mathrm{~T}$ cell line constitutively expressing Cas9, and performed T7 endonuclease I (T7EI) 
assay following gRNA transfection. All the synthetic gRNAs showed higher efficiency than IVTgRNA, in particular, the chemically modified gRNAs (Fig. 3A). Although addition of a cap to 5'-end of IVT-gRNA was detrimental for obtaining efficient genome-editing, uncapped IVT-gRNA maintained 10\% efficiency (Fig. 3A). When the Cas9 was expressed following transfection of chemically modified Cas9 mRNA which is immune silent, IVT-gRNA, full-length unmodified and full-length modified gRNAs showed efficiency of 10\%, 14\% and 22\%, respectively, similar pattern as in 293T-Cas9 cells (Fig. 3B). However, when we preassembled the gRNAs with Cas9 protein to RNP, IVT-gRNA and full-length unmodified gRNA presented same efficiency (Fig. 3C). Notably, preassembly did not remove the gap of efficiency between full-length unmodified and modified gRNA (Fig. 3C) - a phenomenon that has been noticed previously (Hendel et al. 2015). To investigate influences of RNA sensors on the efficiency of genome editing, we generated RIG-I, PKR and MDA5 knockout cell lines derived from the HEK293T-Cas9 cells. In all tested knockout and wild-type 293T cells, chemically modified full-length gRNA consistently had higher gene disruption efficiency than its unmodified counterpart indicating factors other than the innate RNA-sensing proteins being responsible for lower gene editing by unmodified full-length gRNA (Fig. 3A, 3D-F). In RIG-I knockout, but not $P K R$ and MDA5 knockout cell line, IVT-gRNA showed same level of gene disruption efficiency as unmodified full-length gRNA reflecting competition between Cas9 and RIGI protein to bind to IVT-gRNA (Fig. 3D-F).

A

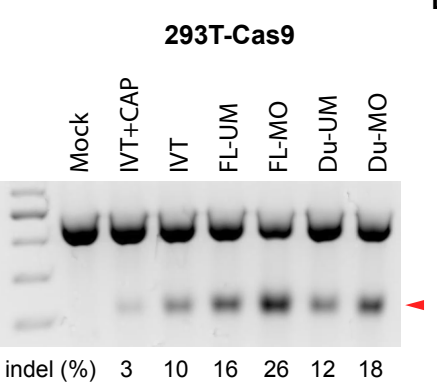

D
B

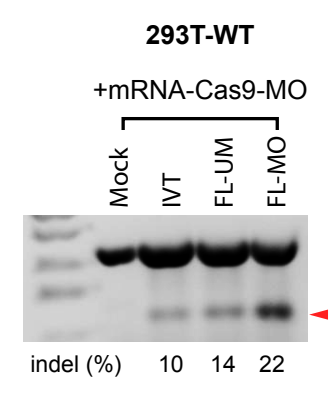

$\mathrm{E}$

C

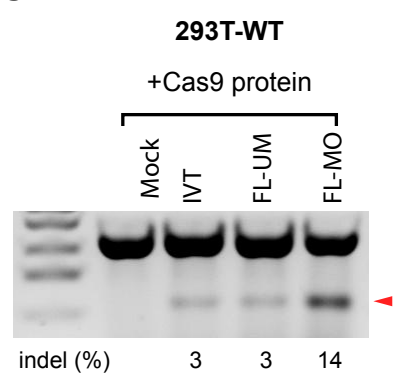

$\mathrm{F}$

293T-Cas9/MDA5 KO

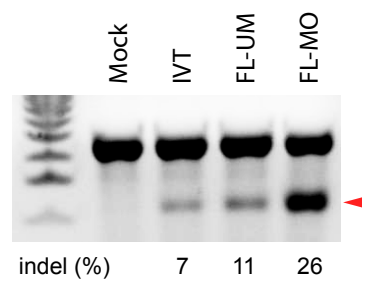

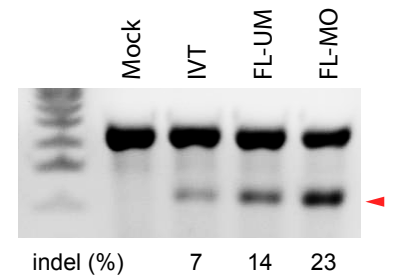

Fig. 3. The nuclease activity associated with gRNA variants examined by T7EI assay. (A) Comparison of nuclease activity of a variety of gRNA in a Cas9-expressing 293T line. From left to 
1 right, capped in vitro transcribed gRNA (IVT+CAP), in vitro transcribed gRNA (IVT), synthetic fulllength gRNA with (FL-MO) or without chemical modification (FL-UM), synthetic and duplexed gRNA with (Du-MO) or without chemical modification (Du-UM). (B) Nuclease activity of IVT, FLUM and FL-MO gRNA in wild-type 293T cells. The Cas9 were provided from modified Cas9 mRNA (Cas9-mRNA-MO) which does not elicit innate immune response. (C) Gene disruption induced by gRNA variants preassembled with recombinant Cas9 proteins in 293T WT cells. (D-F) Nuclease activity of IVT, FL-UM and FL-MO gRNA in RIG-I, PKR and MDA5 knockout 293T cell lines. All the knockout lines consistently express Cas9. All gRNAs target to CCR5 locus.

\section{The antiviral effects of dual functioning gRNA and the mechanism}

Many viruses still cause significant threat to human health. For instance, there is no sterilizing cure for HSV and hepatitis B virus (HBV) infection. CRISPR system has been adapted for treatment of DNA virus (Seeger and Sohn 2014; Seeger and Sohn 2016; van Diemen et al. 2016). So far, the antiviral function of CRISPR has been purely relying on the nuclease activity (Soppe and Lebbink 2017), which makes it less attractive in practice due to the challenge to deliver CRISPR to every infected cell. Innate immune sensing of virus leads to expression and secretion of IFNs which further induce expression of hundreds of IFN-stimulated genes (ISGs) encoding antiviral proteins - thereby protecting both infected and non-infected bystander cells (Paludan 2015). However, viruses have developed mechanisms to escape detection by host cells. For instance, HSV-1 encodes several proteins that antagonize intracellular nucleic acid sensors (Beachboard and Horner 2016; Christensen and Paludan 2017). HSV-1 initiate infection in epithelial cells and subsequently establish latency in sensory neurons. To study the antiviral action of immunostimulatory gRNAs, we chose keratinocytes which are of the epithelial cell linage, and an important target for HSV-1 infection. First, we designed gRNAs targeting HSV-1 and examined whether they induced IFNBI expression in HaCaT keratinocytes. We found IVT-gRNA induced robust IFNB1 expression in HaCaT cells while no IFNB1 production from the FL-MO counterpart (Fig. 4A) using a HSV-1 UL8 targeting gRNA sequence of which the nuclease ability had been characterized previously (van Diemen et al. 2016). Interestingly, however, HSV-1 infection in HaCaT did not induce detectable IFNBI and MXI mRNA nor type I IFN bioactivity (Supplemental_Fig_S4A-C). As unmodified Cas9 mRNA also induces cytokines (Supplemental_Fig_S2), we examined whether we could maximize immune responses by 
gRNA has a tendency to induce more IFNB1 production when combined with unmodified Cas9 mRNA instead of modified one (Fig. 4B). Notably, the immune maximized combination did not affect the efficiency of gene disruption when testing on endogenous CCR5 locus (Fig. 4C). Next, we compared the antiviral activity of different CRIPSR combination against HSV1-GFP by measuring the percentage of GFP positive cells. We found the immune silent combination - full-length unmodified gRNAs and modified Cas9 mRNA only reduced GFP modestly but significantly compared to mock-treated control cells (Fig. 4D). In contrast, in the immune maximized group, IVTgRNA and unmodified Cas9 mRNA strongly suppressed viral gene expression (Fig. 4D). Therefore, we chose the immune maximized combination for further investigation. We evaluated the antiviral activity of two gRNAs targeting to HSV-1 UL8 and UL29 gene, respectively. On day 1, both IVTgRNAs suppressed HSV1-GFP infection significantly (Fig. 4E and Fig. 4F, and Supplemental_Fig_S4D and 4E, left column). On day 2, the virus infection did progress even in the presence of IVT-gRNA (Fig. 4G and Fig. 4H, and Supplemental_Fig_S4E, right column and 4F). However, the IVT gRNA-treated cells preserved intact colony structure in sharp contrast to the mock which failed to form large colonies highlighted by dash lines (Fig. 4H and Supplemental_Fig_S4E, right column) indicating the IVT-gRNAs provide bystander protection to the neighbouring nontransfected cells. In addition, the robust antiviral effects from IVT-gRNA was replicated by the plaque assay using the supernatants from IVT-gRNA treated cells from day 1 (Fig. 4I and Supplemental_Fig_S4G). The bystander versus genome-editing-mediated protection from IVTgRNA can be distinguished by microscopy, since not all cells are transfected with the delivered RNAs. We compared the antiviral activity of immunostimulatory and immune silent RNAs. Interestingly, only the immunostimulatory RNA, but not the immune silent RNAs led to protection of large areas of the infected cell culture (Supplemental_Fig_S4H). To examine whether type I IFNs participated in IVT-gRNA-mediated antiviral responses, we generated a IFNAR2 knockout HaCaT line which does not respond to type I IFNs (Supplemental_Fig_S5A, B). In this setting, the immunostimulatory RNAs, i.e. IVT-gRNA and unmodified Cas9 mRNA induced relatively strong antiviral activity but to a much less extent than in wild-type cells (Fig. 4J). Importantly, IFNAR2 KO cells, failed to provide bystander protection to non-transfected cells as no large intact colony was found (Fig. 4K). Collectively, these data demonstrate that the type I IFN system provides potent adding benefits to the CRISPR-mediated antiviral activity. 
A

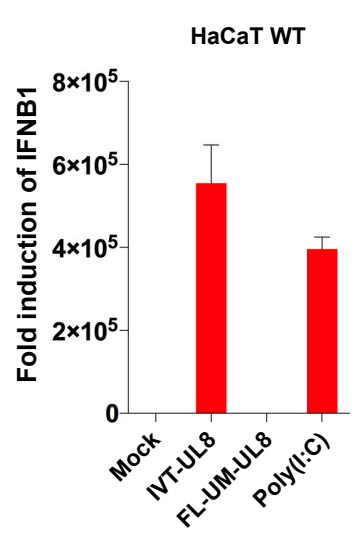

$E$

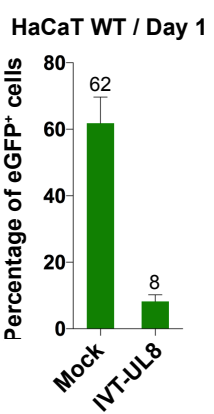

I

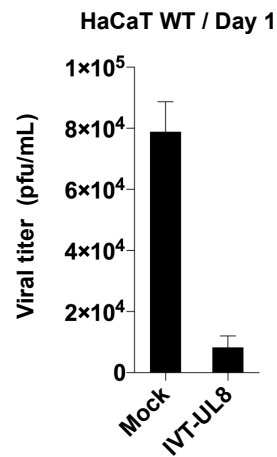

$\mathrm{F}$
B
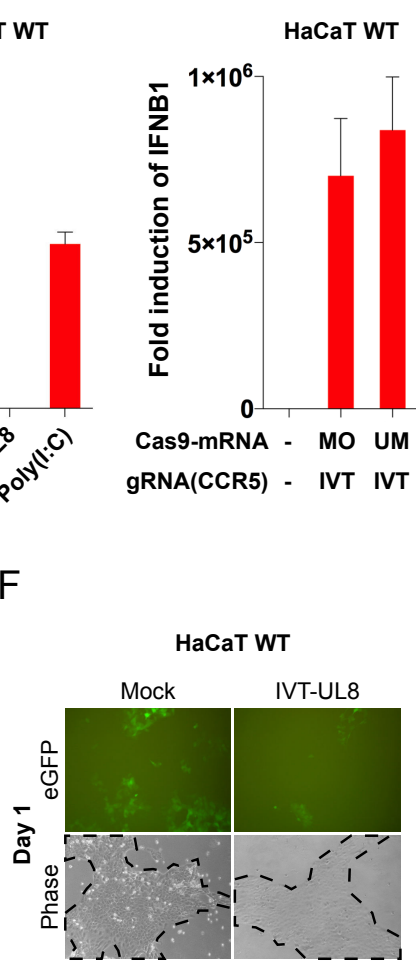

$J$

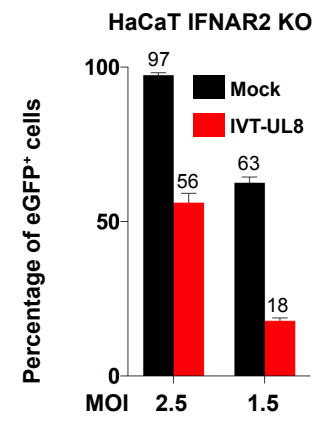

C
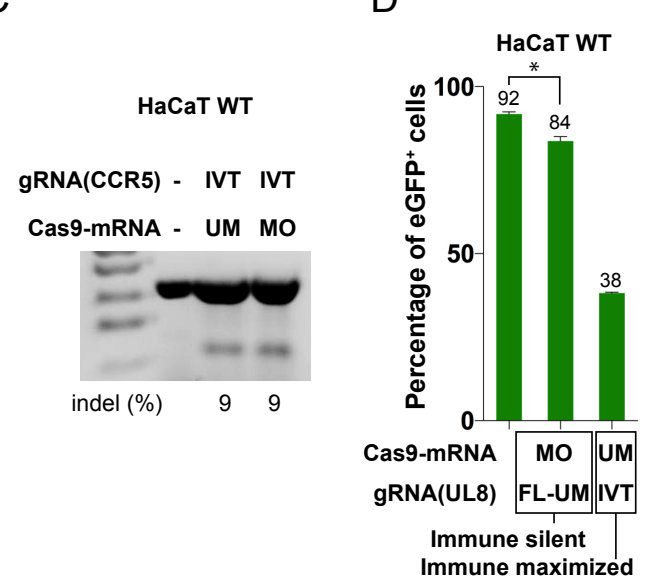

$\mathrm{H}$

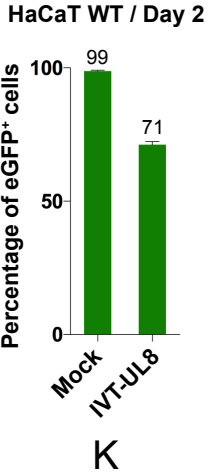

HaCaT WT

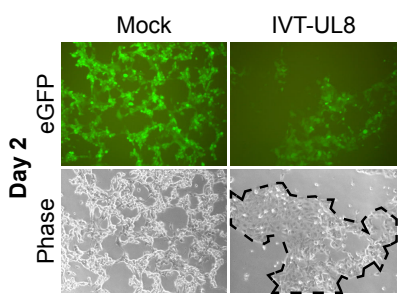

HaCaT IFNAR2 KO

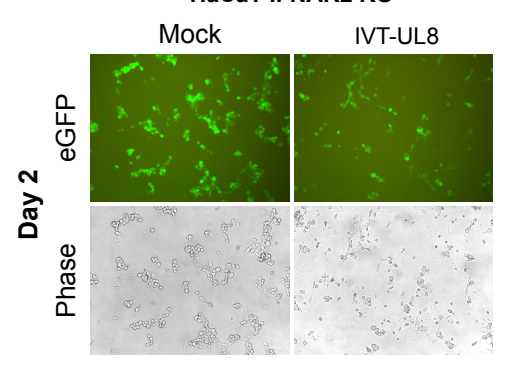

2 Fig. 4. The antiviral effect of IVT-gRNA in vitro. (A) Fold induction of IFNBI by HSV-1 UL8 3 targeting in vitro transcribed (IVT-UL8) and full-length unmodified gRNAs (FL-UM-UL8) and 4 Poly(I:C) in HaCaT cells. (B) Fold induction of IFNB1 by a combination of Cas9-mRNA-MO and 5 IVT-gRNA, and a combination of Cas9-mRNA-UM and IVT-gRNA in HaCaT cells. The gRNA targets to endogenous CCR5 locus. (C) T7EI assay analysis gene disruption at CCR5 locus by IVT-

7 gRNA supplemented with unmodified or modified Cas9 mRNA. Percentage of gene disruption (indel) 8 is shown at the bottom. (D) Antiviral effects by 'immune silencing' combination (Cas9-mRNA-MO 9 and FL-UM) and 'immune maximized' combination (Cas9-mRNA-UM and IVT-gRNA). The gRNA targets to HSV-1 UL8 gene. (E-I) Antiviral effects of IVT-gRNA in HSV1-GFP infected HaCaT cells. 
1 CRISPR was provided by transfection of IVT-gRNA and unmodified Cas9 mRNA $1 \mathrm{~h}$ after HSV1-

2 GFP infection (MOI=1.5). The gRNA targets to HSV-1 UL8. (E) Flow cytometry analysis the 3 percentage of $\mathrm{eGFP}^{+}$cells $24 \mathrm{~h}$ after infection. (F) Representative fluorescent and phase contrast 4 photograph of CRISPR treated cells from (E). (G) Flow cytometry analysis the percentage of eGFP 5 cells $48 \mathrm{~h}$ after infection. (H) Representative fluorescent and phase contrast photograph of CRISPR treated cells from (G). (I) Plaque assay analysis of infectious virus in supernatants harvested from(E).

7 (J) Antiviral effects of IVT-gRNA in HaCaT cells knock out of IFNAR2 indicated by percentage of $8 \mathrm{eGFP}^{+}$cells $24 \mathrm{~h}$ after HSV1-GFP infection (MOI=1.5 or 2.5). The gRNA targets to HSV-1 UL8. 9 The Cas9 was provided by unmodified Cas9 mRNA. HaCaT cells were treated same as (E). (K)

A

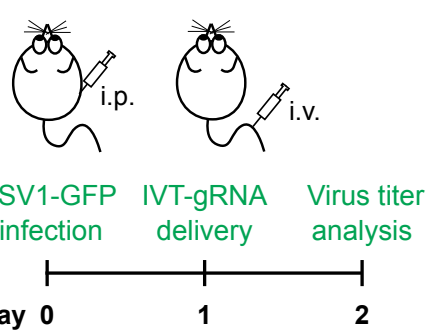

B

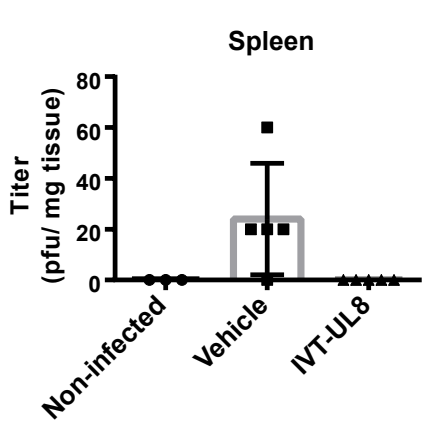

C

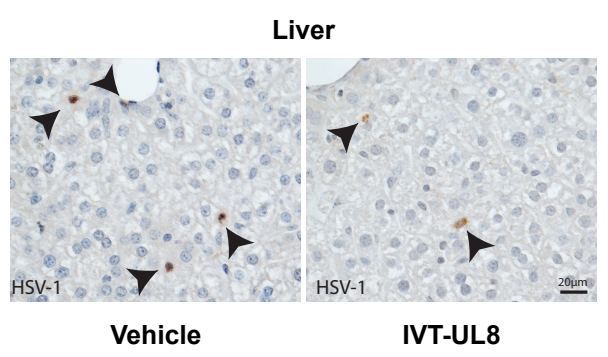


Fig. 5. The antiviral effect of IVT-gRNA in vivo. A. Treatment scheme. Cas9+ mice were infected i.p. with HSV1-GFP. One day later, the mice were treated with IVT-gRNA encapsulated into nanoparticles and delivered i.v. Spleens and livers were harvested $24 \mathrm{~h}$ post infection for further analysis. B. Virus titers in the spleens were determined by plaque assay. C. Liver sections were stained with anti-HSV-1 antibody. Black arrows indicate HSV-1 positive cells. Scale bar $=20 \mu \mathrm{m}$.

\section{Discussion}

In a recent publication by Kim et al., the authors investigated innate immune sensing of IVT-gRNA and found that 1) IVT gRNA induces type I IFN, 2) IVT-gRNA is sensed by RIG-I, a $D D X 58$ encoding protein, 3) Removing IVT gRNA 5'-triphosphate group silences immune response(Kim et al. 2018). While our study confirms the major findings in above-mentioned paper, we provide more detailed investigation regarding mechanism of gRNA sensing. While Kim et al. are interested in avoiding innate immune sensing of IVT-gRNA for gene editing in T cells(Kim et al. 2018), we are interested in exploring the dual function of IVT-gRNA - gene editing and immune stimulating - for antiviral purpose.

Briefly, in this report we have evaluated the immune properties of a variety of commonly used gRNAs and find that only in vitro transcribed gRNA induce potent innate immune responses while synthetic gRNAs do not, irrespective of chemical modifications. In agreement with previous reports on in vitrotranscribed RNA and siRNAs (Kato et al. 2006; Marques et al. 2006), we show that the RIG-I/MAVS pathway is largely responsible for sensing of IVT-gRNAs. We demonstrate that in vitro transcribed gRNA is much more efficient in suppressing HSV-1 infection than synthetic gRNA due to the dual ability to mediate both immune activation and gene disruption. We further demonstrate IVT-gRNA efficiently suppressing HSV-1 in vivo. The antiviral activity may be further strengthened by adding 5 ' triphosphate to the synthetic chemically modified gRNA to enhance the nuclease activity or using improved delivery methods. Many million people worldwide are persistently infected with viruses. For instance, approximately 400 million people are infected with HBV. The lack of clearance is at least partially contributed by lack of immunological DNA sensing in hepatocytes (but with competent RNA sensing pathway) and failure to induce type I IFN (Sato et al. 2015; Thomsen et al. 2016). Based on the presented current proof-of-concept study, we therefore propose that dual ability IVT-gRNAs have therapeutic potential for antiviral treatment against a range of viruses including HSV-1 and HBV. This urges further exploration. 


\section{Methods}

\section{Mice}

The CRISPR/Cas9 knock-in mice constitutively express Cas9 endonuclease (B6J.129(Cg)Igs $2^{\text {tm1.1(CAG-cas9*)Mmw } / J) ~ w e r e ~ o b t a i n e d ~ f r o m ~ T h e ~ J a c k s o n ~ L a b o r a t o r y . ~ A l l ~ e x p e r i m e n t s ~ w e r e ~ c a r r i e d ~}$ out at the University of Aarhus and approved by Danish government authorities. In the infected groups, mice were infected with HSV1-GFP $\left(10^{6} \mathrm{PFU} /\right.$ mouse $)$ at 1-month age by IP injection. The infected mice were either tail vein injected with vehicle (phosphate buffered saline) or IVT-gRNA (40 $\mu \mathrm{g} /$ mouse) complexed with in vivo-jetPEI ${ }^{\circledR}-$ Gal (Polyplus).

\section{Cells lines}

To generate 293T cell line knockout of $P K R$, a gRNA sequence (5'-ttatccatggggaattacat-3') targeting to PKR was cloned into lentiCRISPRv2 puro plasmid (Addgene plasmid \# 98290). The acquired gRNA plasmid was then used to produce lentiviral vector for infection of wild-type 293T cells. The puromycin resistant clones was expanded and verified for knockout by Sanger sequencing of multiple bacteria clones. 293T knockout of RIG-I (Zhu et al. 2014) and MDA5 are kind gifts from Veit Hornung (Institute for Clinical Chemistry and Clinical Pharmacology, University of Bonn, Germany). A Cas9 expression cassette was introduced to RIG-I and MDA5 knockouts by infection of a lentiviral vector encoding Cas9 gene. To generate THP1 knockout of TRIF which functions in the TLR3 signaling pathway, we designed a gRNA (5'-gatgaggcccgaaaccggtg-3') and cloned into the lentiCRISPRv2 puro (Addgene plasmid \#98290). Lentiviral vector was then packaged and used to infected THP1 cells. Four days after infection, cells were plated at a dilution sufficient to obtain single cell clones. These clones were expanded into larger cultures where they were validated by Western blot analysis (Supplemental_Fig_S3B). To generate HaCaT knockout of IFNAR2 which encodes a subunit of the IFNAR heteromer, a guide sequence (5'-caccggtatttcatatgattcgcc-3') was cloned into the lentiCRISPRv2 puro plasmid (Addgene plasmid \# 98290). The HaCaT cells were transfected with the plasmid using the Lipofectamine 2000 (Invitrogen) according to the manufacturer's protocol. Twenty-four hours post transfection, the cells were seeded in a dilution sufficient to obtain single cell clones after the puromycin selection. The puromycin selection $(2 \mu \mathrm{g} / \mathrm{mL})$ was initiated $48 \mathrm{~h}$ post transfection and continued for $72 \mathrm{~h}$. Hereafter, single cell clones were expanded and validated by Sanger sequencing of bacteria clones and functional analysis (Supplemental_Fig_S5A and B).

\section{Cell cultures and transfection}


1 THP-1 and pDC-gen2.2 cells were maintained in RPMI 1640 medium (Lonza). 293T, HaCaT cells and their knockout cells were maintained in DMEM (Lonza). Both RPMI and DMEM were supplemented with 10\% fetal calf serum (FCS, Sigma-Aldrich), 2 mM L-glutamine (Sigma-Aldrich) $100 \mathrm{U} / \mathrm{mL}$ penicillin, $100 \mathrm{lg} / \mathrm{mL}$ streptomycin (Gibco). To boost cytokine production, THP-1 cells were differentiated into macrophage-like cells with $150 \mathrm{nM}$ phorbol 12-myristate 13-acetate (PMA) (Sigma) before experiment. pDC-gen2.2 cells were cultured on MS-5 irradiated feeder cells. The cells were transfected using Lipofectamine 2000 (ThermoFisher Scientific) for most experiments unless specially indicated where cells were transfected with RNAi/MAX (ThermoFisher Scientific) and Viromer RED (Lipocalyx). Poly(I:C) was purchased from InvivoGen. DsDNA is a 60-mer oligonucleotide derived from the HSV-1 genome.

$1.5 \times 10^{5}$ THP-1 derived macrophage-like cells were transfected with different variants of gRNAs or mRNA-Cas9. $6 \mathrm{~h}$ after transfection, the cells were harvested for total RNA extraction using the High Pure RNA Isolation kit (Roche). $10^{5} \mathrm{HaCaT}$ cells were infected with HSV1-GFP at a MOI of 2.5 or transfected with $2.5 \mu \mathrm{g}$ of IVT-gRNA or poly(I:C), and harvested for RNA extraction $16 \mathrm{~h}$ later. All the cytokines were measured with the TaqMan RNA-to-Ct 1-step kit (Applied Biosystems) using on the Aria Mx Real Time PCR System, and was normalized to Act $\beta$. The used TaqMan gene expression assays were IFNB1 (Hs01077958_s1), TNFA (Hs00174128_m1), CCL5 (Hs00174575_m1), IL-6 (Hs00985639_m1), CXCL10(Hs01124252_g1), MX1 (Hs00895608_m1), CCL2 (Hs00234140_m1), and $A C T B$ (Hs00357333_g1).

\section{HEK-Blue Assay}

The level of bioactive type I IFN in supernatants from mock, IVT-gRNA, poly(I:C) or HSV1-GFP treated cells was determined using HEK-BlueTM IFN-alpha/beta cells (Invivogen), according to manufactures instructions, by monitoring the activation of the ISGF3 pathway. HEK-Blue cells has a functioning type I IFN signalling pathway since they are constructed to stably express the human STAT2 and IRF9 genes. Furthermore, the cells are designed with the secreted embryonic alkaline phosphatase (SEAP) encoding gene under control of the IFN-alpha/beta inducible promotor from ISG54. Thus, upon type I IFN stimulation, the HEK-Blue cells secreted SEAP which is detected using absorbance microplate readers (BioTek). 
293T-Cas9 were transfected with gRNAs alone and 293T cells were co-transfected with gRNAs and Cas9 mRNA or recombinant protein using Lipofectamine 2000 (ThermoFisher Scientific). $48 \mathrm{~h}$ after transfection, the cells were harvested and incubated with QuickExtract (Epicentre). The yields were used as templates for PCR amplification using Phusion Master Mix (ThermoFisher Scientific). The PCR products were incubated with $1 \mu$ T7EI nuclease (NEB) in the presence of NEB buffer 2 at $42^{\circ} \mathrm{C}$ for $30 \mathrm{~min}$ after denaturation and re-annealing. The cleavage products were separated on a $1.5 \%$ agarose gel and stained with ethidium bromide. The percentage of indels was determined as previous description (Cai et al. 2014; Cai et al. 2016). The primers used to amplify CCR5 locus are: forward primer 5'-aaaacagtttgcattcatggagggc-3' and reverse primer 5'-agaagcctataaaatagagccctgt-3'.

\section{Flow cytometry and fluorescent microscope analysis}

$\mathrm{HaCaT}$ cells were seeded on 12-well plate at a density of $10^{5} /$ well and subjected to HSV1-GFP infection with $\mathrm{MOI}=1.5$ or 2.5 as indicated. The cells were subsequently transfected with corresponding gRNA and Cas9 mRNA $1 \mathrm{~h}$ after infection. The GFP expression and cell morphology were recorded by fluorescence microscopy on $24 \mathrm{~h}$ (day 1) or $48 \mathrm{~h}$ (day 2) after infection.

\section{Virus plaque assay}

$\mathrm{HaCaT}$ cells were seeded on 12-well plate at a density of $10^{5} /$ well and infected with HSV1-GFP at a MOI of 1.5. IVT-gRNA and unmodified Cas9 mRNA were transfected $1 \mathrm{~h}$ later. The spleens of mice were homogenized with steel beads (Qiagen) in Tissuelyser (II) (Qiagen) for $3 \mathrm{~min}$ at frequency (30 $\mathrm{s})$. The supernatants were harvested for viral titration. Briefly, Vero cells were seeded in 5-cm diameter plates in DMEM supplemented with 5\% FCS; on the next day, cells media were replaced with $400 \mu 12 \%$ FCS DMEM and $100 \mu 1$ serial dilution of the samples. The dishes were shaken every 10 min for 1 hour before adding $8 \mathrm{~mL} \mathrm{2 \%} \mathrm{FCS} \mathrm{DMEM} \mathrm{containing} \mathrm{2 \%} \mathrm{human} \mathrm{immunoglobulin} \mathrm{(CSL}$ Behring). The plates were further incubated for 2 days. Cells were subsequently stained with $0.03 \%$ methylene blue to allow quantification of plaques.

\section{gRNA and Cas9 RNA}

IVT gRNA in the experiments all in vitro transcribed using HiScribe Kit. The synthetic duplex and full-length gRNAs with or without modifications are all purchased from Synthego. The chemically modified versions of synthetic gRNA contain 2'-O-methyl analogs and 3' phosphorothioate internucleotide linkages at the 5' and 3' terminal three bases of the gRNA. The CCR5 gRNA target to 5'-tactcactggtgttcatctt-3', UL8 gRNA targets to 5'-ggggcagccataccgcgtaa-3' and UL29 gRNA targets to 5'-gcgagcgtacacgtatccc-3'. Both modified and unmodified Cas9 mRNA was purchased 
1 from TriLink. The amount of gRNA used was $2.5 \mu \mathrm{g}$ per transfection and Cas 9 mRNA were $1 \mu \mathrm{g}$ per

2 transfection. The RNP complex was assembled by incubation of gRNA with recombinant Cas9

3 protein (IDT) by 1:1 molar ratio at room temperature for $5 \mathrm{~min}$.

\section{$4 \quad$ Immunohistochemistry}

5 Liver tissues were fixated in $10 \%$ formalin, embedded in paraffin, and cut at $4 \mu \mathrm{m}$. The tissues

6 sections submitted to antigen retrieval in a citrate buffer and blocked in 5\% BSA. The sections were

7 incubated overnight with primary antibody for HSV2 (1/500 dilution, B0116; Dako) and the stain

8 was visualized with appropriate secondary antibody and HRP. Hematoxylin was used to stain cell

9 nuclei.

\section{Statistical analysis}

11 Data are presented as mean \pm SD in all experiments $(n \geqslant 3)$. Student's t-tests were performed as 12 indicated (* indicates statistical significance, $\mathrm{P}<0.05)$.

\section{3 \\ Acknowledgement}

14 We thank Line Reinert, Anja Bille Bohn and Charlotte Christie Petersen to help in collecting the flow cytometry data. The work was supported by a grant from The Lundbeck Foundation (R198- 2015$171)$ and the Novo Nordisk Foundation (NNF16OC0022084).

\section{Author contributions}

Y.C. and S.R.P. conceived the study and designed the experiments; Y.C., A.K., S.J.W., and M.K.T. performed experiments; Y.C., A.K., S.J.W., M.K.T. and S.R.P. analyzed data; Y.C. and S.R.P. wrote the manuscript.

\section{Disclosure declaration}

The authors declare no conflict of interest. 


\section{References}

Beachboard DC, Horner SM. 2016. Innate immune evasion strategies of DNA and RNA viruses. Curr Opin Microbiol 32: 113-119.

Cai Y, Bak RO, Mikkelsen JG. 2014. Targeted genome editing by lentiviral protein transduction of zinc-finger and TAL-effector nucleases. Elife 3: e01911.

Cai Y, Laustsen A, Zhou Y, Sun C, Anderson MV, Li S, Uldbjerg N, Luo Y, Jakobsen MR, Mikkelsen JG. 2016. Targeted, homology-driven gene insertion in stem cells by ZFN-loaded 'all-in-one' lentiviral vectors. Elife 5.

Chew WL, Tabebordbar M, Cheng JK, Mali P, Wu EY, Ng AH, Zhu K, Wagers AJ, Church GM. 2016. A multifunctional AAV-CRISPR-Cas9 and its host response. Nat Methods 13: 868-874.

Christensen MH, Paludan SR. 2017. Viral evasion of DNA-stimulated innate immune responses. Cell Mol Immunol 14: 4-13.

Cong L, Ran FA, Cox D, Lin S, Barretto R, Habib N, Hsu PD, Wu X, Jiang W, Marraffini LA et al. 2013. Multiplex genome engineering using CRISPR/Cas systems. Science 339: 819-823.

Dever DP, Bak RO, Reinisch A, Camarena J, Washington G, Nicolas CE, Pavel-Dinu M, Saxena N, Wilkens $A B$, Mantri $S$ et al. 2016. CRISPR/Cas9 beta-globin gene targeting in human haematopoietic stem cells. Nature 539: 384-389.

Goubau D, Schlee M, Deddouche S, Pruijssers AJ, Zillinger T, Goldeck M, Schuberth C, Van der Veen AG, Fujimura T, Rehwinkel J et al. 2014. Antiviral immunity via RIG-I-mediated recognition of RNA bearing 5'-diphosphates. Nature 514: 372-375.

Hendel A, Bak RO, Clark JT, Kennedy AB, Ryan DE, Roy S, Steinfeld I, Lunstad BD, Kaiser RJ, Wilkens $A B$ et al. 2015. Chemically modified guide RNAs enhance CRISPR-Cas genome editing in human primary cells. Nat Biotechnol doi:10.1038/nbt.3290.

Hornung V, Ellegast J, Kim S, Brzozka K, Jung A, Kato H, Poeck H, Akira S, Conzelmann KK, Schlee M et al. 2006. 5'-Triphosphate RNA is the ligand for RIG-I. Science 314: 994-997.

Jinek M, Chylinski K, Fonfara I, Hauer M, Doudna JA, Charpentier E. 2012. A programmable dualRNA-guided DNA endonuclease in adaptive bacterial immunity. Science 337: 816-821.

Jinek M, East A, Cheng A, Lin S, Ma E, Doudna J. 2013. RNA-programmed genome editing in human cells. eLife 2: e00471.

Kato H, Takeuchi O, Sato S, Yoneyama M, Yamamoto M, Matsui K, Uematsu S, Jung A, Kawai T, Ishii $\mathrm{KJ}$ et al. 2006. Differential roles of MDA5 and RIG-I helicases in the recognition of RNA viruses. Nature 441: 101-105.

Kim S, Koo T, Jee HG, Cho HY, Lee G, Lim DG, Shin HS, Kim JS. 2018. CRISPR RNAs trigger innate immune responses in human cells. Genome Res doi:10.1101/gr.231936.117.

Komor AC, Kim YB, Packer MS, Zuris JA, Liu DR. 2016. Programmable editing of a target base in genomic DNA without double-stranded DNA cleavage. Nature 533: 420-424.

Lassig C, Matheisl S, Sparrer KM, de Oliveira Mann CC, Moldt M, Patel JR, Goldeck M, Hartmann G, Garcia-Sastre A, Hornung V et al. 2015. ATP hydrolysis by the viral RNA sensor RIG-I prevents unintentional recognition of self-RNA. Elife 4.

Maeder ML, Linder SJ, Cascio VM, Fu Y, Ho QH, Joung JK. 2013. CRISPR RNA-guided activation of endogenous human genes. Nat Methods doi:10.1038/nmeth.2598.

Mali P, Aach J, Stranges PB, Esvelt KM, Moosburner M, Kosuri S, Yang L, Church GM. 2013. CAS9 transcriptional activators for target specificity screening and paired nickases for cooperative genome engineering. Nat Biotechnol doi:10.1038/nbt.2675. 
Marques JT, Devosse T, Wang D, Zamanian-Daryoush M, Serbinowski P, Hartmann R, Fujita T, Behlke MA, Williams BR. 2006. A structural basis for discriminating between self and nonself doublestranded RNAs in mammalian cells. Nat Biotechnol 24: 559-565.

Mogensen TH. 2009. Pathogen recognition and inflammatory signaling in innate immune defenses. Clin Microbiol Rev 22: 240-273, Table of Contents.

Niu Y, Shen B, Cui Y, Chen Y, Wang J, Wang L, Kang Y, Zhao X, Si W, Li W et al. 2014. Generation of Gene-Modified Cynomolgus Monkey via Cas9/RNA-Mediated Gene Targeting in One-Cell Embryos. Cell doi:10.1016/j.cell.2014.01.027.

Paludan SR. 2015. Activation and Regulation of DNA-Driven Immune Responses. Microbiol Mol Biol Rev 79: 225-241.

Perez-Pinera P, Kocak DD, Vockley CM, Adler AF, Kabadi AM, Polstein LR, Thakore PI, Glass KA, Ousterout DG, Leong KW et al. 2013. RNA-guided gene activation by CRISPR-Cas9-based transcription factors. Nat Methods doi:10.1038/nmeth.2600.

Pichlmair A, Schulz O, Tan CP, Naslund TI, Liljestrom P, Weber F, Reis e Sousa C. 2006. RIG-I-mediated antiviral responses to single-stranded RNA bearing 5'-phosphates. Science 314: 997-1001.

Piras F, Riba M, Petrillo C, Lazarevic D, Cuccovillo I, Bartolaccini S, Stupka E, Gentner B, Cittaro D, Naldini $L$ et al. 2017. Lentiviral vectors escape innate sensing but trigger p53 in human hematopoietic stem and progenitor cells. EMBO Mol Med doi:10.15252/emmm.201707922.

Sanchez David RY, Combredet C, Sismeiro O, Dillies MA, Jagla B, Coppee JY, Mura M, Guerbois Galla $M$, Despres $P$, Tangy $F$ et al. 2016. Comparative analysis of viral RNA signatures on different RIG-I-like receptors. Elife 5: e11275.

Sato S, Li K, Kameyama T, Hayashi T, Ishida Y, Murakami S, Watanabe T, lijima S, Sakurai Y, Watashi $\mathrm{K}$ et al. 2015. The RNA sensor RIG-I dually functions as an innate sensor and direct antiviral factor for hepatitis B virus. Immunity 42: 123-132.

Seeger C, Sohn JA. 2014. Targeting Hepatitis B Virus With CRISPR/Cas9. Mol Ther Nucleic Acids 3: e216.

Seeger C, Sohn JA. 2016. Complete Spectrum of CRISPR/Cas9-induced Mutations on HBV cccDNA. Mol Ther 24: 1258-1266.

Soppe JA, Lebbink RJ. 2017. Antiviral Goes Viral: Harnessing CRISPR/Cas9 to Combat Viruses in Humans. Trends Microbiol doi:10.1016/j.tim.2017.04.005.

Takizawa H, Fritsch K, Kovtonyuk LV, Saito Y, Yakkala C, Jacobs K, Ahuja AK, Lopes M, Hausmann A, Hardt WD et al. 2017. Pathogen-Induced TLR4-TRIF Innate Immune Signaling in Hematopoietic Stem Cells Promotes Proliferation but Reduces Competitive Fitness. Cell Stem Cell 21: 225-240 e225.

Thomsen MK, Nandakumar R, Stadler D, Malo A, Valls RM, Wang F, Reinert LS, Dagnaes-Hansen F, Hollensen AK, Mikkelsen JG et al. 2016. Lack of immunological DNA sensing in hepatocytes facilitates hepatitis B virus infection. Hepatology 64: 746-759.

van Diemen FR, Kruse EM, Hooykaas MJ, Bruggeling CE, Schurch AC, van Ham PM, Imhof SM, Nijhuis M, Wiertz EJ, Lebbink RJ. 2016. CRISPR/Cas9-Mediated Genome Editing of Herpesviruses Limits Productive and Latent Infections. PLoS Pathog 12: e1005701.

Vojta A, Dobrinic P, Tadic V, Bockor L, Korac P, Julg B, Klasic M, Zoldos V. 2016. Repurposing the CRISPR-Cas9 system for targeted DNA methylation. Nucleic Acids Res 44: 5615-5628.

Zhu J, Zhang Y, Ghosh A, Cuevas RA, Forero A, Dhar J, Ibsen MS, Schmid-Burgk JL, Schmidt T, Ganapathiraju MK et al. 2014. Antiviral activity of human OASL protein is mediated by enhancing signaling of the RIG-I RNA sensor. Immunity 40: 936-948. 
bioRxiv preprint doi: https://doi.org/10.1101/282558; this version posted March 15, 2018. The copyright holder for this preprint (which was not certified by peer review) is the author/funder. All rights reserved. No reuse allowed without permission. 